\title{
Assessing the Phase Accuracy of ePIE Reconstructions of Crystalline Materials
}

\author{
Arthur M. Blackburn ${ }^{1 *}$ and Robert A. McLeod ${ }^{2}$ \\ ${ }^{1}$ University of Victoria, Centre for Advanced Materials and Related Technology, Victoria, BC, Canada. \\ ${ }^{2}$ Entropy Reduction Algorithmics, Victoria, BC, Canada. \\ *Corresponding author: ablackbu@uvic.ca
}

Advances in high-speed direct-electron pixelated detectors are allowing greater use of scanning diffractive techniques in electron microscopy. One such technique is ptychography, which permits a quantitative determination of the change in phase and magnitude imparted on the transmitted electron wavefunction, with the extended ptychographic iterative engine (ePIE) algorithm [1] showing much promise through providing exceptionally high spatial resolution reconstructions. However, familiarization and greater understanding of the dose sensitivity for this algorithm, which employs a single scattering phase object approximation in its most widely used commercial implementation [2], and its potential artifacts and inaccuracies is required if one is to apply this technique to wide ranging samples. Furthermore, understanding is required to enable minimal electron dose sampling and rapid sample throughput. To investigate this, we used a newly developed python control system for the electron microscope and reconstruction process. Interpretation of the results has been aided by multi-slice simulations.

Looking at example polycrystalline nanoparticles, as expected effects are seen from the employed ePIE algorithm not accounting for multiple scattering and have limited capabilities at low electron doses. In the reconstructions from experimental data, this manifests as changes in the perceived lattice that stabilizes with increasing electron dose, as shown in the example experimental reconstructions in Figure 1. However, within the reconstructions we see that the overall low spatial frequency trends and reconstructed phase shift quantity remains stable over a wide range of electron dose. This is in broad agreement with models made using multi-slice forward scattering with QSTEM code [3], and ePIE reconstruction from the generated diffraction patterns, incorporating shot noise (Figure 2).

Additionally, there is dependence on the sample orientation, thickness and illumination conditions, in agreement with earlier work $[4,5]$, which makes the selection of the optimum condition for mixed crystalline and amorphous samples challenging. With small convergence angle beam conditions which are suitable for amorphous materials, crystallites will likely be inaccurately reconstructed in the absence of overlap between diffraction orders. This is illustrated through simulated reconstructions of silicon $5 \mathrm{x}$ $5 \mathrm{~nm}$ cuboids of varying thickness. Example results in Figure 3, using a worst case situation for dynamical scattering of illumination directly along a zone axis (100), show that it is possible to gain visually plausible but quantitatively inaccurate reconstructions with small convergence angles (Fig. 3(a)), as seen for example through comparison to reconstructions made from datasets generated using larger convergence angles (Fig. 3(b)). However, even in this condition there is limited accuracy, with deviations increasing with sample thickness (Fig. 3(c)).

Finally, though the accuracy of the beam positioning at the non-monotonic spacings required for ePIE is also known to affect the reconstructions, we show that experimentally it can be well controlled, alleviating need for positional annealing algorithms [6]. In summary, though developments in hardware and software for ptychography now make this versatile technique accessible [7], we highlight that care must be taken in selecting appropriate experimental conditions and in interpreting the resulting data. 
References:

[1] AM Maiden and JM Rodenburg, Ultramicroscopy 109 (2009), p. 1256.

[2] $\pi$-Box, from Phase Focus Ltd, Sheffield, United Kingdom.

[3] C Koch, PhD Thesis, Arizona State University (2002).

[4] C Liu, T Walther and JM Rodenburg, Ultramicroscopy 109 (2009), p. 1263.

[5] T Plamann and JM Rodenburg, Acta Crystallogr Sect A 54 (1998), p. 61.

[6] AM Maiden et al., Ultramicroscopy 120 (2012), p. 64.

[7] The collaboration with Hitachi High Technologies Canada in developing the software and incorporating the hardware on the HF3300V TEM used here is acknowledged with sincere thanks.
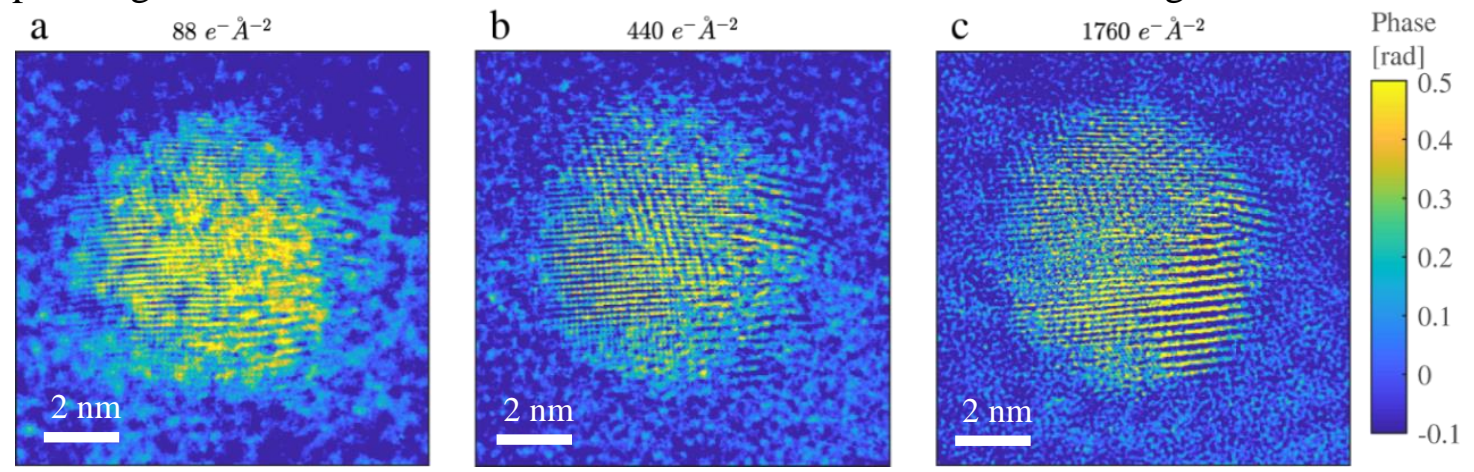

Figure 1. Phase reconstructions created using the ePIE algorithm of a gold particle on carbon film at electron dose of 88, 440 and $1760 \mathrm{e} / \AA^{2}(\mathrm{a}-\mathrm{c})$. Beam conditions: $200 \mathrm{keV}$ energy, current $0.81 \mathrm{pA}$, probe diameter $\sim 3 \mathrm{~nm}$, with $75 \%$ overlap, convergence half-angle $3.7 \mathrm{mrad}$, with diffraction patterns collected for 1,5 , and $20 \mathrm{msec}(\mathrm{a}, \mathrm{b}, \mathrm{c})$ on a $256 \times 256$ pixel Medipix $3 \mathrm{RX}$.
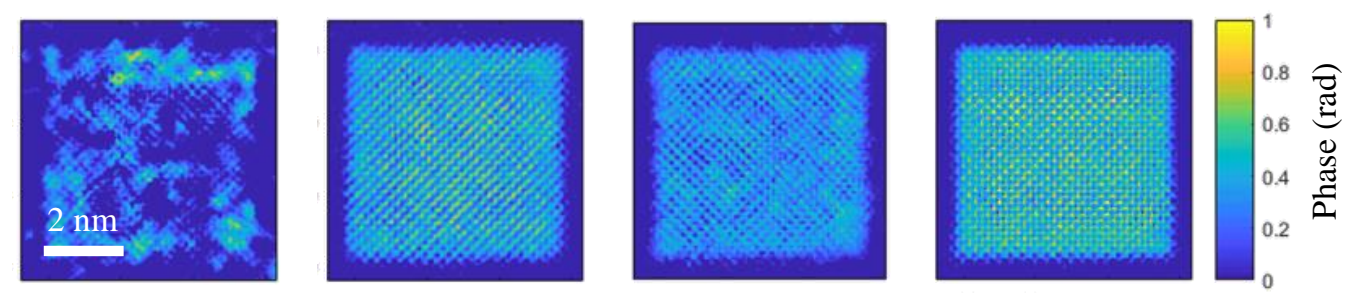

Figure 2. Simulated reconstructions of $2.5 \mathrm{~nm}$ thickness Si100 at doses of (from left to right) 10, 50, 100 and $500 \mathrm{e} / \AA^{2}$.

$\mathbf{a}$

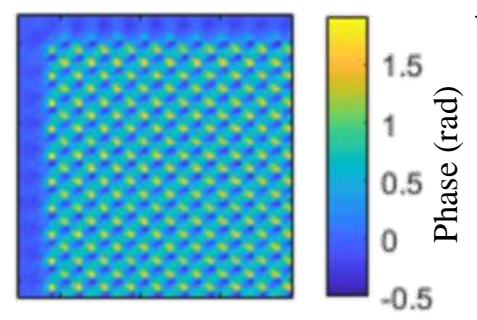

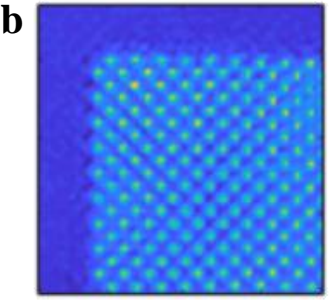

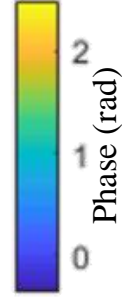

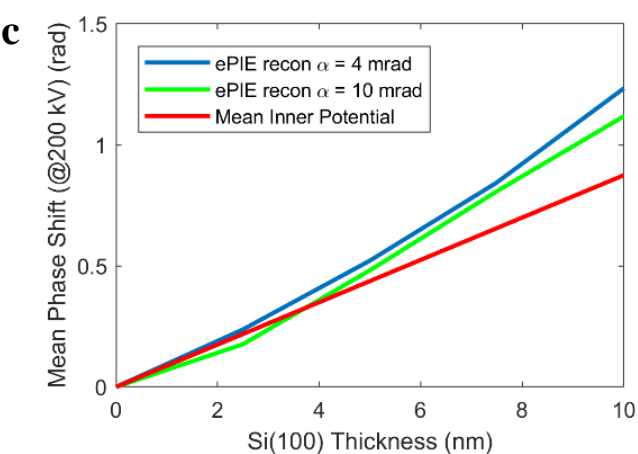

Figure 3. Simulated reconstructions of Si100 cuboids formed (a) $4 \mathrm{mrad}$ (b) $10 \mathrm{mrad}$ illumination convergence angles (200 kV beam). While the former condition is generally inappropriate and leads to inaccurate phase information, general images features remain. (c) The variation of average phase with respect to thickness of the cuboids, compared to a simple mean inner potential model of the phase shift. 